\title{
Cromosomas politénicos de Drosophila sp. nov. ¿Especie primitiva del grupo de Drosophila repleta?
}

\author{
Karina Betancourt \\ Laboratorio de Genética Evolutiva, Escuela de Ciencias Biológicas \\ Pontificia Universidad Católica del Ecuador, Quito. karybetancourt@yahoo.com
}

Recibido: 03, 04, 2012; aceptado: 22, 09, 2012

\begin{abstract}
RESUMEN.- E1 presente trabajo es un reporte de lo hallado en el análisis de los cromosomas politénicos de Drosophila sp. nov. Se realizó placas citológicas de las glándulas salivales de 18 larvas de tercer estadío y se construyó su mapa citológico. En la secuencia cromosómica de esta especie se encontraron las seis inversiones correspondientes a la Primitiva I: Xa, Xb, Xc, 2a, 2 b y 3 b, localizadas en los cromosomas X, 2 y 3 respectivamente. Los cromosomas 4, 5 y 6 muestran una secuencia conservada similar a la hipotética Primitiva I. No se detectó la presencia de alguna inversión paracéntrica, a parte de las de la Primitiva I, en el patrón de bandeo de los cromosomas politénicos de la nueva especie. Los resultados de esta investigación sugieren que Drosophila sp. nov. podría ser una especie primitiva del grupo repleta y probablemente haya dado origen a otras especies miembros de los subgrupos del grupo repleta.
\end{abstract}

PALABRAS CLAVE: Cromosomas politénicos, Drosophila sp. nov., grupo repleta, inversiones paracéntricas, Primitiva I.

ABSTRACT.- This research is the result of polytene chromosome analysis in Drosophila sp. nov. Cytological plaques and maps were made from salivary glands of 18 third-stage larvae. Six inversions corresponding with Primitive I were found in this species' chromosomic sequence: Xa, Xb, Xc, 2a, 2b and 3b, localized in chromosomes X, 2 and 3. Chromosomes 4, 5 and 6 show a conserved sequence similar to the hypothetic Primitive I. Besides Primitive I, there was no evidence for paracentric inversion in the banding pattern of polytene chromosomes of this species. These results suggest that Drosophila sp. nov. is a primitive species of the repleta group and may have given origin to other species within this group.

KEYWORDS: Drosophila sp. nov., paracentric inversions, polytene chromosomes, Primitive I, repleta group.

\section{INTRODUCCIÓN}

Una de las radiaciones de drosophilideos más grandes ocurridas en el Nuevo Mundo es la radiación virilis-repleta cuya historia evolutiva se inició en los trópicos del Viejo Mundo; posteriormente el linaje ancestral de la radiación virilis-repleta arribó a los trópicos del Nuevo Mundo (Throckmorton, 1982; Throckmorton, 1975).
El grupo repleta proviene de al menos un linaje de la radiación inicial ocurrida en los trópicos del Viejo Mundo, es probable que en el neotrópico el origen del grupo haya sido en lo que hoy se conoce como México (Throckmorton, 1975).

La mayoría de especies del grupo repleta son formas desérticas y los cactus se convirtieron en su fuente principal de alimentación 
y refugio para su reproducción, esto podría indicar que la diversificación de las especies del grupo ocurrió ampliamente junto a la aparición de los desiertos en América (Morán y Fontdevila, 2005; Wasserman, 1992; Wasserman, 1982a y b; Throckmorton, 1975).

Además es considerado uno de los grupos más grandes dentro del género Drosophila ya que reúne a casi un centenar de especies que están distribuidas en seis subgrupos: mulleri, hydei, mercatorum, repleta, fasciola e inca (Mafla, 2005a; Ranz et al., 1997).

Los parientes más cercanos al grupo repleta incluyen a los grupos: aureata, cannalinea, castanea y dreyfusi, son especies de bosque, probablemente miembros primitivos del grupo, y conforman la sección repleta. La radiación repleta contiene además al grupo mesophragmatica, estos junto con las especies de los grupos: melanica, peruviana, virilis y robusta forman la rama de la radiación virilis-repleta (Wasserman, 1992; Wasserman, 1982 a y b; Throckmorton, 1982).

Las especies de los grupos que conforman la sección repleta citológicamente están relacionadas con los miembros del grupo repleta, a través de una secuencia conocida como Primitiva I (Wasserman, 1992). La primitiva I es una especie hipotética que reúne una secuencia que surgió a partir de los estudios citológicos realizados por Wasserman, 1960 en varias especies de drosophilideos pertenecientes al grupo repleta. Dicha secuencia incluye seis inversiones: tres localizadas en el cromosoma $\mathrm{X}$ (Xa, Xb y Xc); dos en el cromosoma 2 (2a, $2 b)$; y una en el cromosoma 3 (3b).

La importancia de la Primitiva I es que dicha secuencia es considerada central en la evolución citológica del grupo repleta pues a partir de esta evolucionaron tres grandes líneas filéticas. La primera llevó a la evolución de los subgrupos repleta y mercatorum, la segunda hacia el subgrupo hydei y la tercera al subgrupo mulleri (Wasserman, 1992). Una cuarta línea filética fue propuesta en el año 2008, debido a los resultados obtenidos en el estudio de los cromosomas politénicos de Drosophila huanvavilcae Rafael \& Arcos, 1989, que sugerirían una conexión directa del subgrupo inca con la Primitiva I (Romero, 2008; Romero y Mafla, 2008).

El subgrupo inca, creado en 1989 por la Doctora Violeta Rafael, está conformado por tres especies: Drosophila inca Dobzhansky \& Pavan 1943, Drosophila huancavilcae y Drosophila yangana Rafael \& Vela, 2003, estas dos últimas son especies endémicas del Ecuador (Rafael y Vela, 2003; Rafael y Arcos, 1989).

En el mapa cromosómico de D. huancavilcae se identificó la inversión $2 \mathrm{y}^{5}$ en la región central del cromosoma 2 , que no está presente en ningún miembro de otro subgrupo del grupo repleta. Se encontraron, además, las seis inversiones de la Primitiva I (Xabc, 2ab y 3b) (Romero, 2008).

En los cromosomas politénicos de $D$. yangana se reportaron: 1) Las seis inversiones correspondientes a la Primitiva I (Xabc, 2ab y 3b), 2) Dos nuevas inversiones nominadas: $\mathrm{Xl}$ y 31 localizadas en la región distal y central de los cromosomas X y 3 respectivamente, $y$ 3) No se encontró la inversión $2 y^{5}$ hallada en D. huancavilcae (Betancourt, 2010). Indicando que $D$. huancavilcae y $D$. yangana son dos especies emparentadas a través de la Primitiva I (Betancourt, 2010; Romero, 2008; Romero y Mafla, 2008). El mapa cromosómico de $D$. inca no ha sido construido hasta el momento.

El subgrupo hydei está dividido a su vez en dos complejos: complejo hydei que incluye a las especies: Drosophila eohydei Wasserman, 1962, Drosophila neohydei Wasserman, 1962 y Drosophila hydei Sturtevant, 1921 que incluyen en el patrón de bandeo de sus cromosomas politénicos a más de la Primitiva I a la inversión 2z, y complejo bifurca: Drosophila bifurca Patterson \& Wheeler, 1942, Drosophila nigrohydei Patterson \& Wheeler, 1942 que poseen como secuencia estándar a la Primitiva I Wasserman, 1982a y cuyas especies no presentaban diferenciación cromosómica. 
Sin embargo, un estudio realizado en el año 2001 en los cromosomas politénicos de Drosophila novemaristata Dobzhansky \& Pavan, 1943 y de Drosophila guayllabambae Rafael y Arcos, 1988, especie ecuatoriana, miembros del complejo bifurca, reportó la presencia de dos nuevas inversiones, localizadas en la Región Central del cromosoma 2 nominadas: $2 \mathrm{~b}^{10}$ y $2 \mathrm{c}^{10}$ aumentando el número de inversiones paracéntricas en el acervo del grupo repleta (Morán, 2001), donde hasta el momento se han registrado 301 inversiones (Betancourt, 2010).

Por el contrario, los grupos miembros de la radiación virilis (melanica, peruviana, virilis y robusta), no presentan a la Primitiva I como su secuencia central pero han incorporado en su secuencia a una inversión terminal nombrada como $4 \mathrm{e}$ que también está presente en los grupos aureata, dreyfusi, cannalinea y mesophragmatica de la radiación repleta, y no ha sido encontrada ni en los grupos castanea ni en repleta (Wasserman, 1992).

Es difícil decir con certeza que secuencia es ancestral y cuál es derivada (Primitiva I o 4e) pues las inversiones no permiten determinar la dirección de la evolución pero si son determinantes fiables de las relaciones de parentesco ya que constituyen eventos de origen único. Es decir: si dos poblaciones (o especies) tienen la misma inversión, están más cercanamente emparentadas entre sí, que con otras poblaciones (o especies) que no posean dicha inversión (González, 2002; Morán, 2001; Wasserman, 1992).

Las investigaciones de diversidad drosophilidea que se llevan a cabo en el Ecuador permitieron en el año 2011 encontrar una nueva especie de drosófila en la Provincia de Manabí: Drosophila sp. nov. (comunicación oral, Acurio, 2011).

Estudios citológicos preliminares de los cromosomas metafásicos de Drosophila sp. nov. Indican la presencia de 12 cromosomas (5 pares de barras y 1 par puntiforme) similar al cariotipo ancestral encontrado en las espe- cies del grupo repleta. Sin embargo los datos obtenidos hasta el momento no son todavía concluyentes porque se ha encontrado un número importante de placas citológicas en las cuales se ha observado 10 y 8 cromosomas en el cariotipo de esta nueva especie (comunicación oral, Mafla, 2012).

Por otro lado, los primeros análisis morfológicos externos e internos de esta especie la vincularon con las especies del subgrupo inca, pero un examen posterior de la morfología interna de Drosophila sp. nov. la relacionó con el grupo peruensis (comunicación oral, Acurio y Rafael, 2011).

La dificultad de ubicar a Drosophila sp. nov. dentro de un grupo determinado y entender sus relaciones de parentesco hacen importante el análisis y construcción del mapa cromosómico de los cromosomas politénicos de esta nueva especie, y pretende brindar un aporte importante en la comprensión de la misma.

\section{MATERIALES Y MÉTODOS}

El análisis citológico de los cromosomas politénicos se realizó a partir de una muestra de Drosophila sp.nov. que se logró criar en el laboratorio de Genética Evolutiva de la Pontificia Universidad Católica del Ecuador (PUCE). Se realizaron placas citológicas de las glándulas salivales de 18 larvas de tercer estadío y para la preparación de los cromosomas se aplicó el protocolo de preparación de cromosomas politénicos por aplastado ó "squash", (Morán, 2001). La edición de las fotografías tomadas en los meses de febrero, marzo y abril del 2012 y la construcción del mapa cromosómico de Drosophila sp. nov. se realizó con el programa Adobe Photo Shop (V.8.0) (Romero, 2008)

Para nominar la secuencia cromosómica de Drosophila sp.nov. se utilizó la nomenclatura empleada por Wharton, 1942 en la secuencia estándar de Drosophila repleta. Cada cromosoma fue dividido en tres regiones de izquierda a derecha 1) Región Distal, 
2) Región Central y 3) Región Proximal. Las regiones a su vez se dividieron en secciones, nominadas con letras mayúsculas; las sección contiene subsecciones, numeradas del 1 al 7 , el tamaño de la subsección es variable; y finalmente las bandas de cada subsección fueron denominadas con letras minúsculas en orden sucesivo (a,b,c...etc.) (Morán, 2001).

La posición de las inversiones paracéntricas se especificó según los puntos de rotura que indicaron: a) la sección en donde ocurrió el rearreglo, definida con letras mayúsculas, b) las subsecciones donde inició y terminó el segmento invertido, mostrado con números y c) las bandas, en ambos extremos, donde se cortó la inversión, nombrándolas con letras minúsculas (Morán, 2001).

\section{RESULTADOS Y DISCUSIÓN}

Los cromosomas politénicos de Drosophila sp. nov. presentaron seis brazos cromosómicos $(\mathrm{X}, 2,3,4,5$ y 6$)$ unidos a través de sus regiones heterocromáticas formando un cuerpo irregular conocido como cromocentro (Gardner, 1971), que en esta especie fue "difuso" (Figura 1 aquí). Los telómeros de cinco, de los seis, cromosomas (X, 2, 3, 4 y 5) presentaron formas peculiares que facilitaron su identificación y mostraron semejanza con los telómeros de la Primitiva I.

El cromosoma $X$ fue el más fácil de reconocer debido a la forma de su telómero semejante a un "clavel". El telómero del cromosoma 2 de Drosophila sp. nov. presentó una forma particular similar a un "balón", mientras que el del cromosoma 3 se parecía a una "copa ensanchada". La forma de "jarrón" del telómero del cromosoma 4 lo hizo muy fácil de distinguir y el cromosoma 5 resaltó en casi todas las placas cromosómicas debido a la forma de "copa alargada" de su telómero.

El cromosoma seis fue el más pequeño y presentó mayor dificultad para encontrarlo pues al parecer se hallaba inmerso en el cromocentro. Se lo encontró únicamente en dos placas citológicas.
Debido a que está constituido en su mayoría por heterocromatina no politeniza, tiene una tasa muy baja de recombinación y no presenta variabilidad genética (Riddle y Elgin, 2006).

En cuanto al mapa cromosómico de Drosophila sp. nov. se construyó usando como estándar a la secuencia Primitiva I que también se utilizó para comparar el patrón de bandeo de cada uno de los cromosomas, se obtuvo los siguientes resultados: El cromosoma $\mathrm{X}$ se dividió en 8 secciones y 30 subsecciones; se encontraron las tres inversiones correspondientes a la Primitiva I: Xa con puntos de quiebre en: D4a- C4g, Xb: F2k- F1a y Xc: G2a- F3a (Wasserman, 1992).

El cromosoma 2 abarcó 8 secciones y 38 subsecciones. Presentó las inversiones 2a: B4e- A3a, y 2b: D1g- C6a de la Primitiva I (Wasserman, 1992).

El cromosoma 3 de la nueva especie incluyó 8 secciones y 36 subsecciones, se encontró la inversión $3 \mathrm{~b}$ correspondiente a la Primitiva I (Wasserman, 1992) con puntos de quiebre: E1a- D3a. La subsección 1b, de la sección $\mathrm{E}$, de este cromosoma resultó difícil de encontrarla extendida en las preparaciones citológicas y siempre se la encontró replegada sobre si mismo lo que hizo imposible utilizar un segmento apropiado para formar parte del mapa citológico construido.

Los cromosomas 4 y 5 de Drosophila sp. nov. presentaron una secuencia conservada similar a la hipotética Primitiva I (Wasserman, 1992). El cromosoma 4 comprendió 8 secciones y 31 subsecciones; el cromosoma 5: 8 secciones y 32 subsecciones.

El cromosoma 6 se dividió en dos secciones y tres subsecciones y exhibió una secuencia similar a la Primitiva I (Wasserman, 1992)

Los resultados obtenidos en esta investigación nos sugieren que Drosophila sp. nov. podría ser una especie primitiva del grupo repleta y probablemente se encuentre relacionada con los subgrupos: inca y/o hydei. 


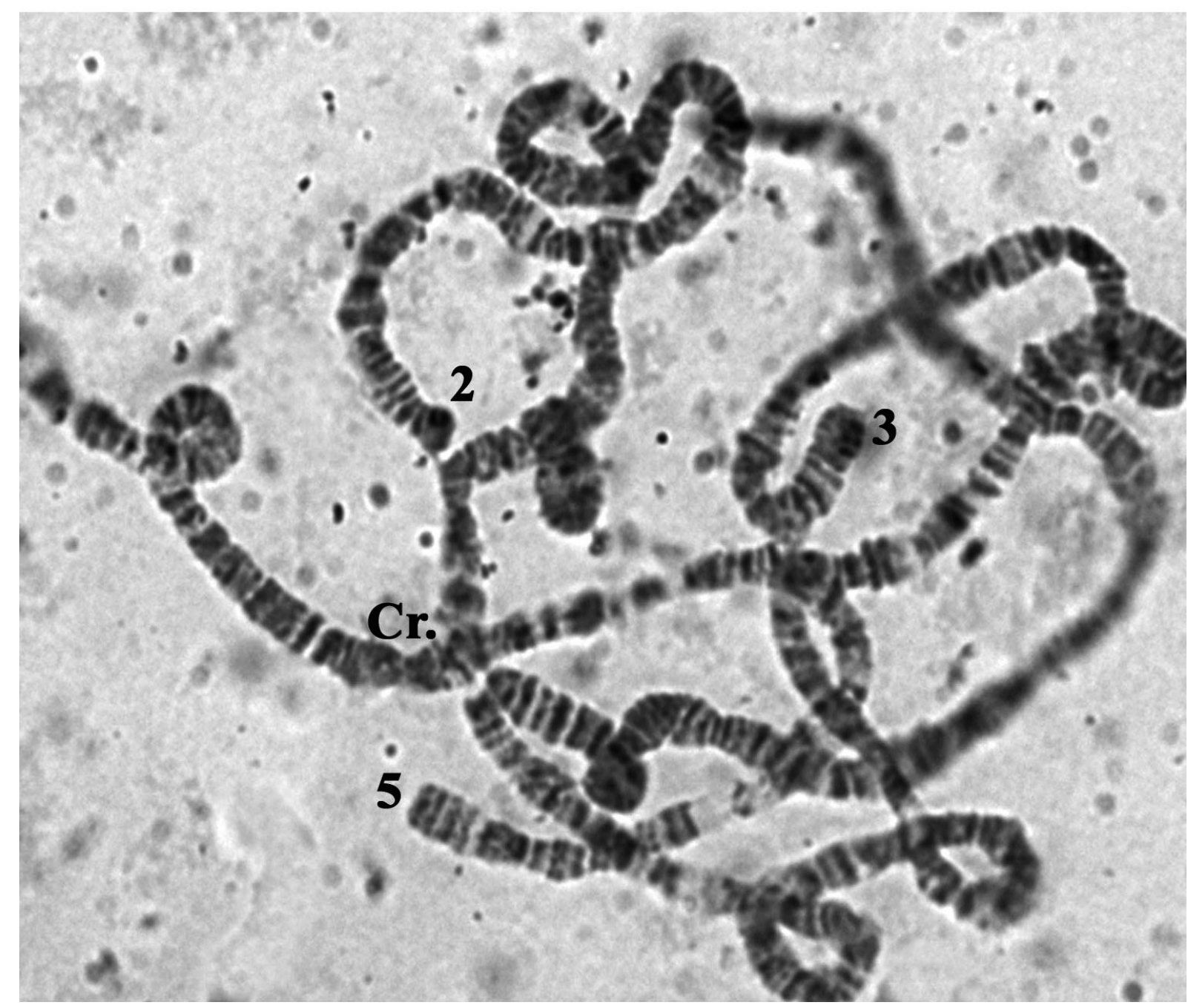

Figura 1. Se observa un núcleo politénico con cinco de los seis brazos cromosómicos unidos al Cromocentro “difuso" (Cr.) de Drosophila sp. nov. Se distinguen en la foto los cromosomas 2, 3 y 5.

El número de seis brazos cromosómicos encontrado en las preparaciones citológicas de los cromosomas politénicos de la especie Drosophila sp. nov son consistentes con un cariotipo ancestral que ha sido encontrado en otras especies del grupo repleta como: Drosophila inca, Drosophila huancavilcae y Drosophila yangana del subgrupo inca (Mafla, 2005b; Mafla, 2008) y en los miembros del subgrupo hydei (Wasserman, 1992).

Además, la ausencia de inversiones paracéntricas extras, a parte de las seis inversiones de la Primitiva I, en el patrón de bandeo de los cromosomas politénicos de esta nueva especie sugiere la probabilidad de que esta sea aquella especie que Wasserman consideró como hipotética y poseedora de la secuencia ancestral conocida como Primitiva I con sus seis inversiones características: Xabc, 2ab y 3b, a partir de la cual podrían haberse originado las cuatro líneas filéticas propuestas por Wasserman, 1992 y Romero y Mafla, 2008.

La relación más cercana que tendría Drosophila sp. nov. podría ser con el subgrupo hydei o con el subgrupo inca pues considerando que la fijación de cada una de las inversiones en un linaje determinado requiere un periodo de tiempo bastante extenso, una por millón de años (Ranz, 1997), los miembros de ambos subgrupos registran la menor tasa de fijación de inversiones paracéntricas comparadas con otras especies del resto de los subgrupos del grupo repleta (Wasserman, 1982a). 


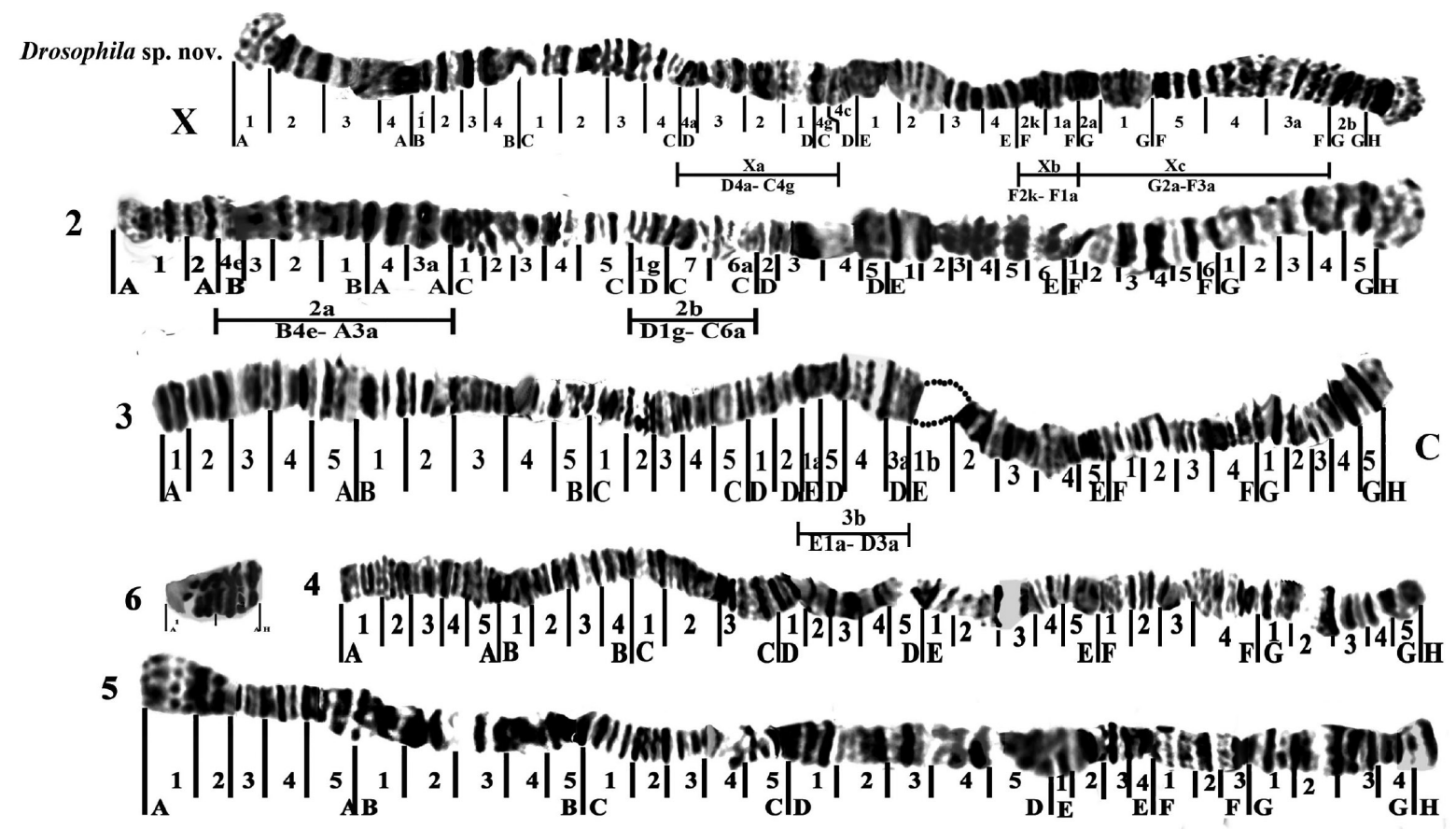

Figura 2. Mapa Cromosómico de Drosophila sp. nov. Se observan los seis cromosomas (X, 2, 3, 4, 5, 6) y las seis inversiones (Xa, Xb, Xc, 2a, 2b y 3b). (C) Cromocentro. La sección en puntos que se observa en el cromosoma 3 corresponde a la subsección $3 \mathrm{~b}$ que no ha sido visible en ninguna placa cromosómica.

Es así que en el subgrupo repleta se encuentran las inversiones $2 \mathrm{i}^{5}, 2 \mathrm{j}^{5}$ y $2 \mathrm{k}^{5}$ (Primitiva IV), los miembros del subgrupo mercatorum tienen a la Primitiva V como secuencia ancestral con las inversiones $2-3 \mathrm{~F}, 2 \mathrm{det}$ y $3 \mathrm{df}$, las especies del linaje mulleri-fasciola tienen a la Primitiva VII como secuencia primordial con las inversiones $2 \mathrm{o}^{2}, 2 \mathrm{e}^{3}$ y $2 \mathrm{l}^{3}$ (Wasserman, 1982a).

Mientras que en el subgrupo inca encontramos las inversiones: $2 \mathrm{y}^{5}$ en $D$. huancavilcae (Romero, 2008; Romero y Mafla, 2008) y $\mathrm{X} 1$ y 31 en D. yangana (Betancourt, 2010) y en el subgrupo hydei las inversiones: $2 \mathrm{~b}^{10}$ y $2 \mathrm{c}^{10}$ en el complejo bifurca y $2 \mathrm{z}$ para el complejo hydei (Wasserman, 1982a).

Es probable también que Drosophila sp. nov. sea la especie basal a partir de la cual se bifurcaron los subgrupos hydei e inca y de esta manera tenga una conexión directa con los dos subgrupos.

En cuanto a la inclusión, de Drosophila sp. nov. en el grupo peruensis debido a simi- litudes encontradas en la genitalia del macho con miembros de dicho grupo, se puede decir que discrepa con el análisis de los cromosomas politénicos realizados en esta especie.

La especie Drosophila atalaia Vilela \& Sene, 1982 que pertenece al grupo peruensis presenta en su cariotipo metafásico tres pares de cromosomas metacéntricos (Vilela y Sene, 1982) que no coinciden con el número de cromosomas encontrado en Drosophila sp. nov. (seis pares), lamentablemente no se han realizado estudios a nivel de cromosomas politénicos en ninguna de las especies del grupo peruensis.

Muller, 1940 y Sturtevant y Novitski, 1941 llegaron a la conclusión de que el cariotipo ancestral del género Drosophila debía estar formado por cinco cromosomas en forma de barra y uno puntiforme ya que es más difícil la formación de dos cromosomas a partir de uno, con la aparición de un nuevo centrómero, que la fusión de dos cromosomas (Ranz et al., 1997). 
De esta manera entonces podríamos decir que $D$. atalaia necesariamente tendría que ser una especie derivada de Drosophila sp. nov. pues debió haber ocurrido fusión cromosómica para que en la actualidad el cariotipos metafásico del de D. atalaia conste de tres pares de cromosomas.

Las discordancias presentadas entre los estudios taxonómicos y citológicos no son una novedad pues en el género Drosophila la mayoría de grupos de especies se han establecido mediante la comparación de las similaridades y diferencias morfológicas observados en cada especie, sin embargo, el estudio de los cromosomas politénicos de las glándulas salivares de los dípteros en muchos de los casos ha reforzado dicha clasificación morfológica pero en otros el análisis de dichos cromosomas a revelado relaciones de parentesco insospechadas a nivel morfológico, tal es el caso de las especies $D$. nannoptera Wheeler MR, 1949, D. acanthoptera Wheeler MR, 1949 y D. pachea Patterson JT \& Wheeler MR, 1942 (Ward y Heed, 2006).

D. nannoptera y D. acanthoptera taxonómicamente eran lo suficientemente distintas como para ser clasificadas en dos diferentes subgéneros, Sophophora y Sordophita respectivamente, posteriormente basados en estudios de la morfología interna realizados por Throckmorton D. nannoptera fue transferida al subgénero Drosophila (Ward y Heed, 2006).

D. pachea fue asignada al subgénero Drosophila y se la relacionó con las especies del grupo repleta, pero Throckmorton encontró mayor similaridad con D. nannoptera (Ward y Heed, 2006).

Sin embargo, al analizar los cromosomas politénicos de las tres especies las similitudes en el número de brazos cromosómicos, patrón de bandeo y en la forma de sus telómeros fueron evidentes. Estas tres especies que originalmente fueron asignadas a tres subgéneros distintos difieren únicamente por cuatro inversiones fijadas. De esta manera las relaciones de parentesco de estas especies siguen siendo inciertas pues necesitan ser re-examinadas morfológicamente (Ward y Heed, 2006).

Este es solo un ejemplo de la disparidad que ocurre entre los análisis morfológicos y cromosómicos del género Drosophila.

Por otro lado, estudios moleculares llevados a cabo en Drosophila sp. nov. en España han descartado la posibilidad de que esta especie pertenezca al grupo peruensis y ha llegado a la conclusión de que es una especie basal de la radiación virilis-repleta (comunicación verbal, Acurio, 2012).

Hay que aclarar que la construcción del mapa cromosómico de Drosophila sp. nov. se llevó a cabo con una muestra reducida de la especie pues al momento de realizar las placas citológicas y tomar las fotografías de los núcleos politénicos de la especie, las cepas de la misma no se habían asentado todavía en el laboratorio y no se contaba con una población estable y suficiente con la cual trabajar, por lo que no se asegura que el ordenamiento génico: Xabc, 2ab y 3 b reportado en esta investigación se cumpla para toda la población y será necesario aumentar la muestra poblacional y hacer más estudios cromosómicos, ecológicos y morfológicos para establecer con mayor exactitud la relación de parentesco de esta especie con los miembros de la radiación virilis- repleta y el grupo peruensis.

Este estudio constituye un aporte al conocimiento de esta nueva especie encontrada en el Ecuador ya que a pesar de haber trabajado con una muestra pequeña, se logró construir un mapa de los cromosomas politénicos de Drosophila sp. nov a la vez que se pudo analizar su patrón de bandeo en busca de nuevas inversiones paracéntricas.

\section{AGRADECIMIENTOS}

A la Pontificia Universidad Católica del Ecuador por la asignación de fondos a través del Proyecto: Cariología Z de nuevas especies de drosófilas del Ecuador. 


\section{REFERENCIAS BIBLIOGRÁFICAS}

Betancourt K. 2010. Cromosomas Politénicos de Drosophila yangana (grupo repleta, subgrupo inca, Díptera, Drosophilidae). Tesis de Licenciatura, Pontificia Universidad Católica del Ecuador. Quito, Ecuador.

Gardner EJ. 1971. Principios de Genética. Segunda edición. Editorial Limusa-Wiley, S.A. México, México. 551 pp.

González PJ. 2002. Evolución Comparada de los Elementos Cromosómicos en el género Drosophila. Título de Doctora en Ciencias Biológicas, Universidad Autónoma de Barcelona. Barcelona, España.

Mafla AB. 2005a. Ciclos de vida y componentes de la aptitud de Drosophila inca y Drosophila yangana (Diptera, Drosophilidae). Iheringia série Zoologia, 95(1):89-91.

Mafla AB. 2005b. Cariotipos Metafásicos de Drosophila inca y Drosophila yangana, subgrupo inca, grupo repleta. Revista Ecuatoriana de Medicina y Ciencias Biológicas, 27(1 y 2): 21-25.

Mafla AB. 2008. Drosophila huancavilcae: Ciclo biológico y cariotipo Metafásico. Revista Ecuatoriana de Medicina y Ciencias Biológicas, 19(1 y 2) 35-39.

Morán T. 2001. Cariología Z y Significado Evolutivo del Fenómeno de Asinápsis Somática en Drosophila novemaristata y Drosophila guayllabambae (Grupo repleta, Subgrupo hydei, Díptera Drosophilidae). Tesis de Licenciatura, Pontificia Universidad Católica del Ecuador. Quito, Ecuador.

Morán T y Fontdevila A. 2005. Phylogeny and molecular evolution of the Drosophila hydei subgroup (Drosophila repleta group) inferred from Xanthine dehydrogenase gene. Molecular Phylogenetics and Evolution, 36 (3): 695-705.

Rafael V y Arcos G. 1989. Subgrupo inca, un nuevo subgrupo del grupo repleta, con Descripción de Drosophila huancavilcae, n.sp. /Díptera, Drosophilidae). Evolución Biológica, 3: 233-243.
Rafael V y Vela D. 2003. Drosophila yangana sp. Un nuevo miembro del grupo repleta, subgrupo inca (Diptera: Drosophilidae). Revista de la Pontificia Universidad Católica del Ecuador, 71: 129-139.

Ranz JM, Segarra C y Ruiz A. 1997. Chromosomal homology and molecular Organization of Muller's elements D and E in the Drosophila repleta species group. Genetics, 145: 281-295.

Riddle NC y Elgin SCR. 2006. The dot chromosome of Drosophila: Insights into chromatin states and their change over evolutionary time. Chromosome Research, 14: 405-416.

Romero G. 2008. Mapa Citológico de los Cromosomas Politénicos de Drosophila huancavilcae (grupo Repleta, subgrupo Inca, Díptera, Drosophilidae. Tesis de Licenciatura, Pontificia Universidad Católica Del Ecuador. Quito, Ecuador.

Romero GC y Mafla AB. 2008. Polytene chromosomes of Drosophila huancavilcae (repleta group, inca subgroup). Drosophila Information Service, 91:182-90.

Throckmorton LH. 1975. The Phylogeny, Ecology and Geography of Drosophila. En: R. C. King (ed). Handbook of Genetics, 3: 421-429. Plenum Publishing Corporation. New York.

Throckmorton LH. 1982. Pathways of Evolution in the Genus Drosophila and the Pounding of the Repleta group. Ecological Genetics and Evolution, 33-37.

Vilela RC y Sene FM. 1982. A new spotted thorax species of the genus Drosophila (Díptera, Drosophilidae) Revista Brasileira de Entomología, 26(3/4): 343-347.

Ward BL y Heed WB. 2006. Chromosome Phylogeny of Drosophila pachea and Related species. American Genetic Association. The journal of Heredity, 248-258.

Wasserman M. 1960. Cytological and Phylogenetic relationships in the repleta group of the genus Drosophila. Proceeding of the National Acadamy of Sciences, 56(6): 842-859. 
Wasserman M. 1982a. Evolution of the repleta group. En: M. Ashburner HL. Carson y JN. Thompson JR. (eds). The Genetics and Biology of Drosophila, 3b: 61-139.

Wasserman M. 1982b. Cytological evolution in the Drosophila repleta species group. Ecological Genetics and Evolution, 49-64.
Wasserman M. 1992. Cytological Evolution of the Drosophila repleta species group. En: CB. Krimbas y JR. Powell (eds). Drosophila inversion polymorphism: 455-552. CR. C Press, Boca Ratón, Florida, U.S.A. 